\title{
Outcomes Associated With Pharmacist- Led Consult Service for Opioid Tapering and Pharmacotherapy
}

\author{
Michelle M. Krichbaum, PharmD; Christine M. Vartan, PharmD, BCPS; Abigail T. Brooks, PharmD, BCPS; and \\ Sandra L. DiScala, PharmD, BCPS
}

Background: In response to the growing opioid epidemic, the US Department of Veterans Affairs created the Opioid Safety Initiative to monitor opioid prescriptions and reduce the risk of opioid misuse through electronic consults (eConsult). The primary objective of this study was to assess the outcomes of the pain pharmacist recommendations for opioid tapering and opioid pharmacotherapy by quantifying the number of recommendations accepted/implemented by the prescribers.

Methods: Data gathered include patient demographics, pain scores, and morphine equivalent daily doses. Additional data included pain regimens, the reason for opioid taper request, the number of actionable recommendations made and implemented, and adverse events (AEs) related to opi- oid dose decrease or taper. A feedback assessment was completed to assess health care provider (HCP) satisfaction with the eConsult service.

Results: We identified 464 eConsults placed with $89 \%$ having at least 1 recommendation accepted/implemented. Eleven patients experienced 32 AEs likely associated with opioid dose decrease or tapering. HCP satisfaction with the pharmacy eConsult service was $83 \%$, with $90 \%$ planning to use the service in the future and would recommend the service to other HCPs.

Conclusions: A pain pharmacy eConsult service managed by pharmacists assisted HCPs with opioid therapy recommendations in a safe and timely manner, reducing risk of overdose secondary to high-dose opioid therapy and with limited patient harms.
Michelle Krichbaum is a Pain Management Clinical Coordinator at Broward Health Medical Center in Fort Lauderdale, Florida. Christine Vartan, Abigail Brooks, and Sandra DiScala are Clinical Pharmacy Specialists, all at West Palm Beach Veterans Affairs Medical Center in Florida

Correspondence: Michelle Krichbaum (m1krichbaum@ browardhealth.org)

Fed Pract. 2021;38(5) Published online May 17. doi:10.12788/fp.0098 n the late 1980s and early 1990s, an emphasis on better pain management led health care professionals (HCPs) to increase prescribing of opioids to better manage patient's pain. In 1991, 76 million prescriptions were written for opioids in the United States, and by 2011, the number had nearly tripled to 219 million. ${ }^{1}$ Overdose rates increased as well, nearly tripling from 1999 to $2014 .{ }^{2}$ Of the 52,404 US deaths from drug overdoses in the in 2015, $63 \%$ involved an opioid. ${ }^{2}$

\section{OPIOID SAFETY INITIATIVE}

In response to the growing opioid epidemic, the US Department of Veterans Affairs (VA) created the Opioid Safety Initiative in 2014. ${ }^{3}$ This comprehensive, multifaceted initiative was designed to improve the care and safety of veterans managed with opioid therapy and promote rational opioid prescribing and monitoring. In 2016 the Centers for Disease Control and Prevention (CDC) issued guidelines for opioid prescriptions, and the following year the VA and the US Department of Defense (DoD) updated the VA/DoD Clinical Practice Guidelines for Opioid Therapy for Chronic Pain (VA/DoD guidelines). ${ }^{4,5}$ After the release of these guidelines, the use of opioid tapers expanded. However, due to public outcry of forced opioid tapering in 2019, the
US Food and Drug Administration updated its opioid labeling requirements to provide clearer guidance on opioid tapers for tolerant patients. $^{6,7}$

As a result, HCPs began to develop various strategies to balance the safety and efficacy of opioid use in patients with chronic pain. The West Palm Beach VA Medical Center (WPBVAMC) in Florida has a Pain Clinic that includes 2 pain management clinical pharmacy specialists (CPSs) with specialized training in pain management, who are uniquely qualified to assess and evaluate medication therapy in complex pain patient cases. These CPSs were involved in the face-to-face management of patients requiring specialized pain care and participated in a pain pharmacy electronic consult (eConsult) service to document pain management consultative recommendations for patients appropriate for management at the primary care level. This formalized process increased specialty pain care access for veterans whose pain was managed by primary care providers (PCPs).

The pain pharmacy eConsult service was initiated at the WPBVAMC in June 2013 to assist PCPs in the management of outpatients with chronic pain. The eConsult service includes evaluation of a patient's electronic health records (EHRs) by CPSs. The eConsult service also provided PCPs 
with the option to engage a pharmacist who could provide recommendations for opioid dosing conversion, opioid tapering, pain pharmacotherapy, or drug screen interpretation, without the necessity for an additional patient visit.

Subsequent to the release of the 2016 CDC (and later the $2017 \mathrm{VA} / \mathrm{DoD}$ ) guidelines recommending reducing morphine equivalent daily dose (MEDD) levels, the WPBVAMC had a large increase in pain eConsult requests for opioid tapering and opioid pharmacotherapy. A 3.4-fold increase in requests occurred in March, April, and May vs the following 9 months, and a nearly 4-fold increase in requests for opioid tapers during the same period. However, the impact of the completed eConsults was unclear. Therefore, the primary objective of this study was to assess the effect of CPS services for opioid tapering and opioid pharmacotherapy by quantifying the number of recommendations accepted/ implemented by PCPs. The secondary objectives included evaluating harms associated with the recommendations (eg, increase in visits to the emergency department [ED], hospitalizations, suicide attempts, or PCP visits) and provider satisfaction.

\section{METHODS}

A retrospective chart review was completed to assess data of patients from the WPBVAMC and its associated communitybased outpatient clinics (CBOCs). The project was approved by the WPBVAMC Scientific Advisory Committee as part of the facility's performance improvement efforts.

Included patients had a pain pharmacy eConsult placed between April 1, 2016 and March 31, 2017. EHRs were reviewed and only eConsults for opioid pharmacotherapy recommendation or opioid tapers were evaluated. eConsults were excluded if the request was discontinued, completed by a HCP other than the pain CPS, or placed for an opioid dose conversion, nonopioid pharmacotherapy, or drug screen interpretation.

Data for analyses were entered into Microsoft Excel 2016 and were securely saved and accessible to relevant researchers. Patient protected health information used during patient care remained confidential.

Demographic data were collected, including age, gender, race, pertinent medical
FIGURE Exclusion Criteria

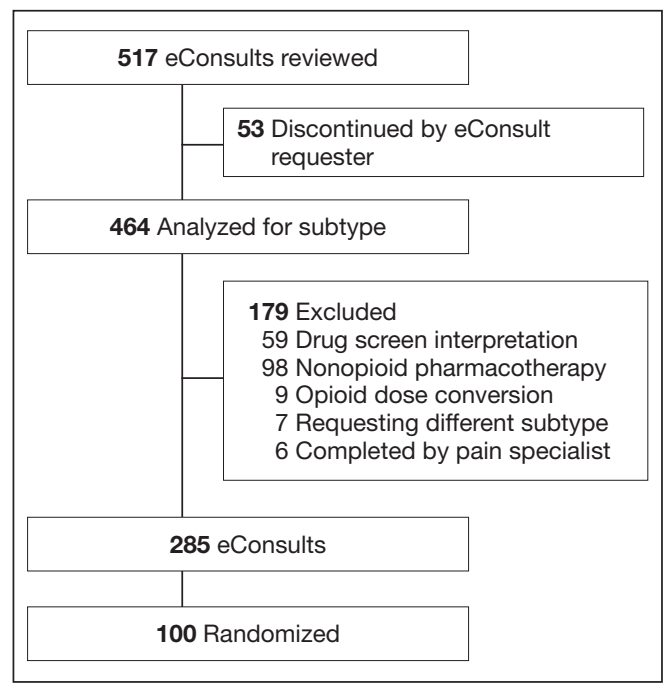

comorbidities (eg, diabetes mellitus, sleep apnea), and mental health comorbidities. Pain scores were collected at baseline and 6 -months postconsult. Pain medications used by patients were noted at baseline and 6 months postconsult, including concomitant opioid and benzodiazepine use, MEDD, and other pain medication. The duration of time needed by pain CPS to complete each eConsult and total time from eConsult entered to HCP implementation of the initial recommendation was collected. The number of actionable recommendations (eg, changes in drug therapy, urine drug screens [UDSs], and referrals to other services also were recorded and reviewed 6 months postconsult to determine the number and percentage of recommendations implemented by the HCP. The EHR was examined to determine adverse events (AEs) (eg, any documentation of suicide attempt, calls to the Veterans Crisis Line, or death 6 month postconsult). Collected data also included new eConsults, the reason for opioid tapering either by HCP or patient, and assessment of economic harms (count of the number of visits to ED, hospitalizations, or unscheduled PCP visits with uncontrolled pain as chief reason within 6 months postconsult). Last, PCPs were sent a survey to assess their satisfaction with the pain eConsult service.

\section{RESULTS}

Of 517 eConsults received from April 1, 2016 to March 31, 2017, 285 (55.1\%) met 
TABLE 1 Patient Characteristics $(\mathrm{N}=100)$

\begin{tabular}{lc} 
Variables & Results \\
\hline Age, mean, y & 61.1 \\
\hline Male, \% & 87 \\
\hline Race, \% & \\
White & 80 \\
African American & 11 \\
Latin/Hispanic & 5 \\
Other & 3 \\
Native Hawaiian or other Pacific Islander & 1 \\
\hline Comorbidities, \%a & \\
Diabetes mellitus & 36 \\
Sleep apnea & 30 \\
Chronic obstructive pulmonary disease & 23 \\
Obesity & 17 \\
Chronic pain & 44 \\
Back pain & 42 \\
Osteoarthritis/degenerative joint disease & 31 \\
Neuropathy/radiculopathy & 19 \\
Neck pain & 16 \\
Headache/migraine & 14 \\
\hline Mental Health Diagnosis, \%a & \\
Depression & \\
Tobacco use & 45 \\
Posttraumatic stress disorder & 35 \\
Anxiety (excluding posttraumatic stress disorder) & 34 \\
Insomnia & 28 \\
Alcohol use disorder & 16 \\
Substance abuse (including cannabis and cocaine) & 15 \\
Bipolar/mood disorder & 13 \\
Opioid abuse/use disordes & 8 \\
apatients may have > 1 condition. & 5 \\
\hline
\end{tabular}

inclusion criteria (Figure). Using a random number generator, 100 eConsults were further reviewed for outcomes of interest.

In this cohort, the mean age was 61 years, $87 \%$ were male, and $80 \%$ were White individuals. Most patients (83\%) had $\geq 1$ mental health comorbidity, and $53 \%$ had $\geq 2$, with depressive symptoms, tobacco use, and/or posttraumatic stress disorder the most common diagnoses (Table 1). Eighty-seven percent of eConsults were for opioid tapers and the remaining 13\% were for opioid pharmacotherapy.

The median pain score at time of consult was 6 on a 10-point scale, with no change at 6 months postconsult. However, $41 \%$ of patients overall had a median 3.3-point drop in pain score, $17 \%$ had no change in pain score, and $42 \%$ had a median 2.6-point increase in pain score.

At time of consult, 24\% of patients had an opioid and benzodiazepine prescribed concurrently. At the time of the initial request, the mean MEDD was $177.5 \mathrm{mg}$ (median, 165; range, 0-577.5). At 6 months postconsult, the average MEDD was $71 \mathrm{mg}$ (median,
90; range, 0-450) for a mean 44\% MEDD decrease. Eighteen percent of patients had no change in MEDD, and 5\% had an increase.

One concern was the number of patients whose pain management regimen consisted of either opioids as monotherapy or a combination of opioids and skeletal muscle relaxants (SMRs), which can increase the opioid overdose risk and are not indicated for long-term use (except for baclofen for spasticity). Thirty-five percent of patients were taking either opioid monotherapy or opioids and SMRs for chronic pain management at time of consult and 28\% were taking opioid monotherapy or opioids and SMRs 6 months postconsult.

\section{Electronic Consults}

Table 2 describes the reasons eConsults were requested. The most common reason was to taper the dose to be in compliance with the CDC 2016 guideline recommendation of MEDD $<90 \mathrm{mg}$, which was later increased to $100 \mathrm{mg}$ by the VA/DoD guideline.

On average, eConsults were completed within a mean of 11.5 days of the PCP request, including nights and weekends. The CPS spent a mean 66.8 minutes to complete each eConsult. Once the eConsult was completed, PCPs took a mean of 9 days to initiate the primary recommendation. This 9-day average does not include 11 eConsults with no accepted recommendations and 11 eConsults for which the PCP implemented the primary recommendation before the CPS completed the consult, most likely due to a phone call or direct contact with the CPS at the time the eConsult was ordered.

A mean 3.5 actionable recommendations were made by the CPS and a mean 1.6 recommendations were implemented within 6 months by the PCP. At least 1 recommendation was accepted/implemented for $89 \%$ of patients, with a mean $55 \%$ recommendations that were accepted/implemented. Eleven percent of the eConsult final recommendations were not accepted by PCPs and clear documentation of the reasons were not provided.

\section{Adverse Outcomes}

In the 6 months postconsult, 11 patients ( 7 men and 4 women) experienced 32 AEs 
(Table 3). Eight patients had 15 ED visits, with 3 of the visits resulting in hospitalizations, 8 patients had 9 unscheduled PCP visits, 1 patient reported suicidal ideation and 2 patients made a total of 4 calls to the Veterans Crisis Line. There were also 2 deaths; however, both were due to end-stage disease (cirrhosis and amyotrophic lateral sclerosis) and not believed to be related to eConsult recommendations.

Eight patients had a history of substance use disorders (SUDs) and 8 had a history of a mood disorder or psychosis. One patient had both SUD and a mood/psychosis-related mental health disorder, including a reported suicidal attempt/ideation at an ED visit and a subsequent hospitalization. A similar number of AEs occurred in patients with decreases in MEDD of 0 to 24\% compared with those that received more aggressive tapers of 75 to $100 \%$ (Table 4).

\section{Primary Care Providers}

Nine patients were reconsulted, with only 1 secondary to the PCP not implementing recommendations from the initial consult. No factors were found that correlated with likelihood of a patient being reconsulted.

Surveys on PCP satisfaction with the eConsult service were completed by 29 of the 55 PCPs. PCP feedback was generally positive with nearly $90 \%$ of PCPs planning to use the service in the future as well as recommending use to other providers.

PCPs also were given the option to indicate the most important factor for overall satisfaction with eConsult service (time, access, safety, expectations or confidence). Safety was provider's top choice with time being a close second.

\section{DISCUSSION}

Most (89\%) PCPs accepted at least 1 recommendation from the completed eConsult, and MEDDs decreased by $60 \%$, likely reducing the patient's risk of overdose or other AEs from opioids. There also was a slight reduction in patient's mean pain scores; however, $41 \%$ had a decrease and $42 \%$ had an increase in pain scores. There was no clear relationship when pain scores were compared with MEDDs, likely giving credence to the idea that pain scores are largely subjective and an unreliable surro-
TABLE 2 Health Care Provider Reasoning for Opioid Taper Request $(n=90)$

\begin{tabular}{lc} 
Reasons & No. (\%) \\
\hline $\begin{array}{l}\text { Guideline recommendation } \\
\begin{array}{l}100 \text { mg morphine equivalent daily dose } \\
\text { Concomitant opioid and benzodiazepine use }\end{array}\end{array}$ & $\begin{array}{r}58(66.6) \\
4(4.6)\end{array}$ \\
\hline Safety & $9(10.3)$ \\
\hline Non-VA opioid use & $6(6.9)$ \\
\hline Patient requested & $5(5.8)$ \\
\hline Cocaine use & $3(3.4)$ \\
\hline Marijuana use & $2(2.3)$ \\
\hline Non-VA benzodiazepines use & $2(2.3)$ \\
\hline Amphetamines use & $1(1.1)$
\end{tabular}

Abbreviation: VA, US Department of Veterans Affairs.

gate marker for assessing effectiveness of analgesic regimens.

Eleven patients experienced AEs, including 1 patient for whom the recommendations were not implemented by the PCP. Eight of the 11 had multiple AEs. One interesting finding was that 7 of the 11 patients with an AE tested positive for unexpected substances on routine UDS or were arrested for driving while intoxicated (DWI). However, only 3 of the 7 had an active SUD diagnosis. With 25\% of the AEs coming from patients with a history of SUD, it is important that any history of SUD be documented in the EHR. Maintaining this documentation can be especially difficult if patients switch VA medical centers or receive services outside the VA. Thorough and accurate history and chart review should ideally be completed before prescribing opioids.

\section{Guidelines}

While the PCPs were following VA/DoD and CDC recommendations for opioid tapering to $<100$ or $90 \mathrm{mg}$ MEDD, respectively, there is weak evidence in these guidelines to support specific MEDD cutoffs. The CDC guidelines even state, "a single dosage threshold for safe opioid use could not be identified." One of the largest issues when using MEDD as a cutoff is the lack of agreement on its calculation. In 2014, Nuckols and colleagues al conducted a study to compare the existing 
TABLE 3 Veterans with Adverse Events following eConsult

\begin{tabular}{|c|c|c|c|c|c|c|c|c|c|}
\hline \multirow[b]{2}{*}{ No. } & \multirow[b]{2}{*}{ Gender } & \multirow[b]{2}{*}{ Taper Reason } & \multirow[b]{2}{*}{$\begin{array}{l}\text { History } \\
\text { SUD }\end{array}$} & \multirow[b]{2}{*}{$\begin{array}{c}\text { Bipolar/Mood/ } \\
\text { Psychosis Diagnosis }\end{array}$} & \multicolumn{3}{|c|}{ Economic Harms, No. } & \multicolumn{2}{|c|}{ Patient Harms, No. } \\
\hline & & & & & $\begin{array}{l}\text { ED } \\
\text { Visits }\end{array}$ & Hospitalizations & $\begin{array}{l}\text { Unscheduled } \\
\text { PCP visits }\end{array}$ & $\begin{array}{l}\text { Suicide } \\
\text { Attempts }\end{array}$ & $\begin{array}{l}\text { Veterans Crisis } \\
\text { Line calls }\end{array}$ \\
\hline 1 & Male & Guidelines & No & No & 5 & 0 & 1 & 0 & 0 \\
\hline 2 & Female & N/A & Alcohol & No & 1 & 0 & 1 & 0 & 0 \\
\hline 3 & Female & N/A & No & No & 1 & 1 & 1 & 0 & 0 \\
\hline 4 & Male & $\mathrm{N} / \mathrm{A}$ & No & Schizo-affective & 0 & 0 & 0 & 0 & 3 \\
\hline 5 & Male & Guidelines & No & Bipolar & 1 & 0 & 0 & 0 & 0 \\
\hline 6 & Male & $\begin{array}{l}\text { UDS violation: } \\
\text { cocaine, BZD1 }\end{array}$ & $\begin{array}{l}\text { Cocaine, } \\
\text { cannabis } \\
\text { alcohol }\end{array}$ & No & 0 & 0 & 1 & 0 & 0 \\
\hline 7 & Female & $\begin{array}{l}\text { UDS violation: } \\
\text { marijuana }\end{array}$ & Opioidsa & No & 2 & 0 & 1 & 0 & 0 \\
\hline 8 & Male & $\begin{array}{l}\text { UDS violation: } \\
\text { cocaine }\end{array}$ & $\begin{array}{l}\text { Opioids }{ }^{b} \\
\text { alcohol } \\
\text { cocaine } \\
\text { cannabis }\end{array}$ & $\begin{array}{l}\text { Mood } \\
\text { disorder }\end{array}$ & 1 & 1 & 1 & 1 & 0 \\
\hline 9 & Male & $\begin{array}{l}\text { UDS violation: } \\
\text { cocaine }\end{array}$ & No & No & 1 & 0 & 2 & 0 & 0 \\
\hline 10 & Female & Guidelines & No & No & 3 & 1 & 1 & 0 & 0 \\
\hline 11 & Male & $\begin{array}{l}\text { Violation: non- } \\
\text { VA narcotics }\end{array}$ & No & $\begin{array}{c}\text { Depression w/ } \\
\text { psychotic features }\end{array}$ & 0 & 0 & 0 & 0 & 1 \\
\hline
\end{tabular}

Abbreviations: BZD, benzodiazepine; SUD, substance use disorder; UDS, urine drug screen; VA, US Department of Veterans Affairs.

aOpioid misuse was inactive at time of eConsult.

${ }^{b}$ Opioid misuse was the only active SUD at time of eConsult, others were listed as inactive.

guidelines on the use of opioids for chronic pain. While 13 guidelines were considered eligible, most recommendations were supported only by observational data or expert recommendations, and there was no consensus on what constitutes a "morphine equivalent." ${ }^{8}$ Currently there is no universally accepted opioid-conversion method, resulting in a substantial problem when calculating a MEDD. ${ }^{9}$ A survey of 8 online opioid dose conversion tools found a $-55 \%$ to $+242 \%$ variation. ${ }^{10}$ As Fudin and colleagues concluded in response to the large variations found in these various analyses, the studies "unequivocally disqualify the validity of embracing MEDD to assess risk in any meaningful statistical way." ${ }^{11}$ Pharmacogenetics, drug tolerance, drug-drug interactions, body surface area, and organ function are patient- specific factors that are not taken into consideration when relying solely on a MEDD calculation. Tapering to lowest func- tional dose rather than a specific number or cutoff may be a more effective way to treat patients, and providers should use the guidelines as recommendations and not a hardline mandate.

At 6 months, 6 patients were receiving no pain medications from the VA, and 24 of the patients were tapered from their opiate to discontinuation. It is unclear whether patients are no longer taking opioids or switched their care to non-VA providers to receive medications, including opioids, privately. This is difficult to verify, though a prescription drug monitoring program (PDMP) could be used to assess patient adherence. As many of the patients that were tapered due to identification of aberrant behaviors, lack of continuity of care across health care systems may result in future patient harm.

The results of this analysis highlight the importance of checking PDMP databases and routine UDSs when prescribing opioids- 
TABLE 4 Changes in MEDD Compared With Changes in Pain Score and Adverse Outcomes $(n=95)$

\begin{tabular}{|c|c|c|c|c|c|c|c|}
\hline $\begin{array}{l}\text { Change in } \\
\text { MEDD, \% }\end{array}$ & $\begin{array}{c}\text { Consults, } \\
\text { No. }\end{array}$ & $\begin{array}{l}\text { Pain Score } \\
\text { Change, } \\
\text { Median }^{\mathrm{a}}\end{array}$ & $\begin{array}{c}\text { ED Visits, } \\
\text { No. }\end{array}$ & $\begin{array}{l}\text { Hospitalizations, } \\
\text { No. }\end{array}$ & $\begin{array}{c}\text { Unscheduled } \\
\text { PCP Visits, } \\
\text { No. }\end{array}$ & $\begin{array}{c}\text { Suicide } \\
\text { Attempts, } \\
\text { No. }\end{array}$ & $\begin{array}{l}\text { Crisis Line } \\
\text { Calls, No. }\end{array}$ \\
\hline Increase & 5 & -1 & 0 & 0 & 0 & 0 & 0 \\
\hline \multicolumn{8}{|l|}{ Decrease } \\
\hline $0-24$ & 25 & 0 & 4 & 2 & 2 & 0 & 3 \\
\hline $25-49$ & 27 & 0 & 6 & 0 & 1 & 0 & 0 \\
\hline $50-74$ & 17 & 1 & 0 & 0 & 0 & 0 & 0 \\
\hline $75-100$ & 21 & 0 & 4 & 1 & 5 & 1 & 1 \\
\hline Total & 95 & N/A & 14 & 3 & 8 & 1 & 4 \\
\hline
\end{tabular}

aNegative number indicates pain score decreased, a positive number indicates pain score increased

Abbreviation: ED, emergency department; MEDD, morphine equivalent daily dose; PCP, primary care provider.

there can be serious safety concerns if patients are taking other prescribed or illicit medications. However, care must be taken; there were 2 instances of patients' chronic opioid prescriptions discontinued by their VA provider after a review of the PDMP showed they had received non-VA opioids. In both cases, the quantity and doses received were small (counts of $\leq 12$ ) and were received more than 6 months prior to the check of the PDMP. While this constitutes a breach of the Informed Consent for long-term opioid use, if there are no other concerning behaviors, it may be more prudent to review the informed consent with the patient and discuss why the behavior is a breach to ensure that patients and PCPs continue to work as a team to manage chronic pain.

\section{Limitations}

The study population was one limitation of this project. While data suggest that chronic pain affects women more than men, this study's population was only $13 \%$ female. Thirty percent of the women in this study had an AE compared with only $8 \%$ of the men. Additional limitations included use of problem list for comorbidities, as lists may be inaccurate or outdated, and limiting the monitoring of AE to only 6 months. As some tapers were not initiated immediately and some taper schedules can last several months to years; therefor, outcomes may have been higher if patients were followed longer. Many of the patients with AEs had increased ED visits or unscheduled primary care visits as the tapers went on and their pain worsened, but the visits were outside the 6-month time frame for data collection. An additional weakness of this review included assessing a pain score, but not functional status, which may be a better predictor of the effectiveness of a patient's pain management regimen. This assessment is needed in future studies for more reliable data. Finally, PCP survey results also should be viewed with caution. The current survey had only 29 respondents, and the 2014 survey had only 10 respondents and did not include CBOC providers.

\section{CONCLUSION}

A pain eConsult service managed by CPSs specializing in pain management can assist patients and PCPs with opioid therapy recommendations in a safe and timely manner, reducing risk of overdose secondary to high dose opioid therapy and with limited harm to patients.

\section{Author disclosures}

The authors report no actual or potential conflicts of interest with regard to this article.

\section{Disclaimer}

The opinions expressed herein are those of the authors and do not necessarily reflect those of Federal Practitioner, Frontline Medical Communications Inc., the US Government, or any of its agencies. This article may discuss unlabeled or investigational use of certain drugs. Please review the complete prescribing information for specific drugs or drug combinations-including indications, contraindications, warnings, and adverse effectsbefore administering pharmacologic therapy to patients.

\section{References}

1. National Institute on Drug Abuse. Increased drug availability is associated with increased use and overdose. Published June 9, 2020. Accessed February 19, 2021. https://www.drugabuse.gov/publications/research-reports /prescription-opioids-heroin/increased-drug-availability 
-associated-increased-use-overdose

2. Rudd RA, Seth P, David F, Scholl L. Increases in drug and opioid-involved overdose deaths - United States, 2010-2015. MMWR Morb Mortal Wkly Rep. 2016;65(50-51):1445-1452. Published 2016 Dec 30 doi:10.15585/mmwr.mm655051e1

3. US Department of Veterans Affairs, Office of Inspector General. Healthcare inspection - VA patterns of dispensing take-home opioids and monitoring patients on opioid therapy. Report 14-00895-163. Published May 14, 2014 Accessed February 2, 2021. https://www.va.gov/oig/pubs NAOIG-14-00895-163.pdf

4. US Department of Veterans Affairs, US Department of Defense, Opioid Therapy for Chronic Pain Work Group. VA/DoD clinical practice guidelines for opioid therapy for chronic pain. Version 3.0. Published December 2017. Accessed February 2, 2021. https://www.va.gov /HOMELESS/nchav/resources/docs/mental-health /substance-abuse/VA_DoD-CLINICAL-PRACTICE -GUIDELINE-FOR-OPIOID-THERAPY-FOR-CHRONICPAIN-508.pdf

5. Dowell D, Haegerich TM, Chou R. CDC Guideline for Prescribing Opioids for Chronic Pain - United States, 2016 [published correction appears in MMWR Recomm Rep. 2016;65(11):295]. MMWR Recomm Rep. 2016;65(1):1-49. Published 2016 Mar 18. doi:10.15585/mmwr.rr6501e1.
6. US Food and Drug Administration. (2019). FDA identifies harm reported from sudden discontinuation of opioid pain medicines and requires label changes to guide prescribers on gradual, individualized tapering. Updated April 17, 2019. Accessed February 2, 2021. https://www.fda .gov/drugs/fda-drug-safety-podcasts/fda-identifies-harm -reported-sudden-discontinuation-opioid-pain-medicines -and-requires-label-changes

7. Dowell D, Haegerich T, Chou R. No Shortcuts to Safer Opioid Prescribing. N Engl J Med. 2019;380(24):2285-2287. doi:10.1056/NEJMp1904190

8. Nuckols TK, Anderson L, Popescu I, et al. Opioid prescribing: a systematic review and critical appraisal of guidelines for chronic pain. Ann Intern Med. 2014;160(1):38-47. doi:10.7326/0003-4819-160-1-201401070-00732

9. Rennick A, Atkinson T, Cimino NM, Strassels SA McPherson ML, Fudin J. Variability in Opioid Equivalence Calculations. Pain Med. 2016;17(5):892-898. doi:10.1111/pme.12920

10. Shaw K, Fudin J. Evaluation and comparison of online equianalgesic opioid dose conversion calculators. Pract Pain Manag. 2013;13(7):61-66.

11. Fudin J, Pratt Cleary J, Schatman ME. The MEDD myth the impact of pseudoscience on pain research and prescribing-guideline development. J Pain Res. 2016;9:153 156. Published 2016 Mar 23. doi:10.2147/JPR.S107794 